\title{
Early or Late Parenteral Nutrition: ASPEN vs ESPEN
}

\author{
Matthew E Cove and Michael R Pinsky \\ University of Pittsburgh Department of Critical Care Medicine: Evidence-Based Medicine Journal Club, edited by Sachin Yende
}

\section{Expanded abstract Citation}

Casaer MP, Mesotten D, Hermans G et al:: Early versus late parenteral nutrition in critically ill adults. $N$ Engl $J$ Med 2011, 365:506-517.

\section{Background}

Controversy exists about the timing of the initiation of parenteral nutrition (PN) in critically ill adults in whom caloric targets cannot be met by enteral nutrition (EN) alone.

\section{Methods}

Objective: To compare early-initiation of PN (European guidelines) with late-initiation (American and Canadian guidelines) in adults who are receiving insufficient enteral nutrition in the intensive care unit (ICU).

Design: Prospective, randomized, controlled, parallelgroup, multicenter clinical trial.

Setting: Seven multidisciplinary ICUs in Belgium.

Subjects: All adults admitted to participating ICUs with a nutritional risk score of 3 or more who did not meet any exclusion criteria.

Intervention: After enrollment, 2312 patients were randomized to receive PN 48 hours after ICU admission (early-initiation) and 2328 patients were randomized to receive $\mathrm{PN}$ on day 8 (late-initiation group). Both groups received early EN using a standardized protocol. PN was continued until EN met $80 \%$ of calorific goals, or when oral nutrition was resumed. It was restarted if enteral or oral feeding fell below $50 \%$ of calculated calorific needs.

Outcomes: Primary end point was the duration of dependency on intensive care, defined as the number of intensive care days and time to discharge from the ICU.

\section{Results}

The median stay in the ICU was one day shorter for the late-initiation group ( 3 v. $4 ; P=0.02$ ). The late-initiation

*Correspondence: coveme@upmc.edu

Department of Critical Care Medicine, 606 Scaife Hall 3550 Terrace Street, University of Pittsburgh, Pittsburgh, PA 15261, USA group had a relative increase, of $6.3 \%$, in the likelihood of being discharged earlier, and alive, from the ICU (hazard ratio 1.06; 95\% confidence interval [CI] 1.00-1,13; $P=0.04)$. Rates of death in the ICU and survival at 90 days were similar between the two groups. The lateinitiation group, as compared to the early-initiation group, had fewer ICU infections $(22.8 \%$ v. $26.2 \%$; $P=0.008)$, less days of renal replacement therapy (7 days (interquartile range [IQR] 3-16) v. 10 days (IQR 5-23); $P=0.008)$ and fewer patients requiring more than 2 days of mechanical ventilation $(36.3 \%$ v. $40.2 \%$; $P=0.006)$.

\section{Conclusions}

Late-initiation of PN was associated with faster recovery and fewer complications, when compared with earlyinitiation.

\section{Trial Registration}

NCT00512122

\section{Commentary}

\section{Background}

Critically ill patients are often unable to feed themselves, and frequently present in a fasting state. Failure to feed these patients eventually guarantees starvation and, if enough time lapses, death. However, it is less certain when fasting becomes starvation. This is important because starvation has deleterious effects, including immune and organ dysfunction [1]. Further, the critically ill patient is already in a catabolic crisis [2].We therefore expect early feeding to be beneficial. Indeed, a metaanalysis in 2001 demonstrated that early EN is associated with less infections (relative-risk 0.45; CI 0.30-0.66; $P<0.001$ ) and shorter hospital stays (mean-reduction 2.2 days; CI 0.81-3.3; $P=0.004$ ). [3] Nutrition guidelines on both sides of the Atlantic agree that early EN is important [4-6].

Achieving calorific goals with EN is challenging in the critically ill. In one study $40 \%$ of ICU patients received no nutrition, while the remaining only received, on average, $58 \%$ of their calorific goals [2]. This is because critically ill patients frequently experience gastroparesis and unnecessary delays waiting for post-pyloric feeding tubes and/or bowel sounds, despite contrary evidence $[7,8]$. 
Supplementing with PN allows practitioners to reach calorific goals earlier, but this maybe harmful. When used alone, PN causes hyperglycemia, hepatocellular injury and immunosuppression [9]. The point where the potential hazards of PN outweigh those of starvation, due failure to reach EN goals, is unclear. On this point European (ESPEN) and North American (ASPEN) nutritional societies disagree. ESPEN recommend commencing PN after 2 days, if patients are receiving less than their targeted EN [5]. In contrast, ASPEN recommend waiting until day $8[4,6]$.

\section{Study}

Casaer and colleagues designed a multicenter trial to challenge this disparity head on. They compared earlyinitiation of PN (day 3) with late-initiation (day 8) [10]. Their primary outcome measure was ICU length of stay (LOS). Both groups received standardized EN, and they were largely successful in achieving uniform EN. To keep fluid intake even, the late-initiation group received 5\% dextrose at the same rate as they would have received PN, were they in the early-initiation group. Recruitment was excellent, with just 5.3\% refusing consent. Exclusion criteria were entirely reasonable, most patients being excluded because they were too young, (age $<18$ ), too well (no central-access, taking oral-nutrition) or not expected to survive (moribund). Patients with a body mass index (BMI) $<17$ were also excluded, withholding $\mathrm{PN}$ in these patients may be considered unethical. In the end, 4640 patients were randomized.

Overall, this study found that delayed PN was superior to early PN. The late-initiation group remained in the ICU one day less (median ICU-LOS; $\mathrm{p}=0.02$ ) which reflected a $6.3 \%$ relative increase in the likelihood of earlier discharge alive from the ICU (hazard ratio, 1.06; CI 1.00-1.13; $P=0.04)$. Secondary outcomes were either the same for both groups, or favored late-initiation. In particular, late-initiation had fewer infections, $(22.8 \% \mathrm{v}$. 26.2\%; $P=0.008$ ) fewer patients requiring $>2$ days of mechanical ventilation $(36.3 \%$ v. $40.2 \% ; P=0.006)$ and less renal replacement therapy (7-days (IQR 3-16) v. 10-days (IQR 5-23); $P=0.008$ ). This translated into an average saving of over $€ 1100(P=0.04)$ per patient. The authors concluded that there is no added benefit to initiating PN earlier and that delayed PN, if needed, was superior.

\section{Critique}

This study has many strengths. It's a large clinical trial with excellent recruitment where both groups are well matched, with balanced fluid intake. Early PN volumes were matched by $5 \%$ dextrose in the late-initiation group. Further, enteral feeding was similar in both groups and both groups received equal micronutrients. This is reflective of a good trial comparing two treatment alternatives, with robust results that allow practice patterns to change.

However, there are some weaknesses that deserve consideration. First, forced early EN and early prokinetic use in all subjects does not match generalized practice patterns, though it might be considered a reasonable baseline for us all to adopt. Second, early placement of post-pyloric tubes is not achievable in many institutions, making the generalizability of these dependent on the ability to place post-pyloric tubes quickly. Third, the study was necessarily unblinded, although physicians making discharge decisions and outcome data-collectors were unaware of treatment allocation. Further, introduction of unblinded bias would be expected to favor earlyinitiation, as the study authors' were directly involved in development of the ESPEN feeding guidelines, which were shown to be inferior.

The results also raise an intriguing question, what about giving these patients no parenteral nutrition at all? This is important because more than $70 \%$ of the lateinitiation group did not receive PN at all, on account of being discharged before day 8 . It is therefore conceivable that the benefits of late-initiation were seen because the majority of patients received no PN. Future trials should investigate delaying parenteral nutrition beyond 8 days, or indeed never giving parenteral nutrition at all, in patients already receiving enteral nutrition.

\section{Recommendation}

Casaer and colleagues are to be commended for conducting a large randomized controlled trail to tackle the current trans-Atlantic guideline disparity for timing of PN. They showed that late-initiation of PN is superior to early-initiation, in patients not meeting targeted EN goals. In the absence of a "no PN" arm we recommend that practitioners wait until at least day 8 before considering $\mathrm{PN}$ in patients with a $\mathrm{BMI}>17$. In patients who are close to $80 \%$ of their calorific goal by day 8 , practitioners should consider that there might be no benefit to adding $\mathrm{PN}$.

\section{Competing interests}

The authors declare that they have no competing interests.

Author contributions

MEC reviewed the background for the manuscript and wrote the original draft and final version of the manuscript.

MRP contributed to planning the outline of the manuscript, revising the text and ensured accuracy of the information contained, as well as editing the draft manuscript.

Acknowledgement

This work was supported in part by NIH grants HL67181 and HL073198.

Published: 22 December 2011 


\section{References}

1. Chandra RK: 1990 McCollum Award lecture. Nutrition and immunity: lessons from the past and new insights into the future. Am J Clin Nutr 1991, 53:1087-1101.

2. Heyland DK, Schroter-Noppe D, Drover JW, Jain M, Keefe L, Dhaliwal R, Day A: Nutrition support in the critical care setting: current practice in canadian ICUs--opportunities for improvement? JPEN J Parenter Enteral Nutr 2003, 27:74-83.

3. Marik PE, Zaloga GP: Early enteral nutrition in acutely ill patients: a systematic review. Crit Care Med 2001, 29:2264-2270.

4. Heyland DK, Dhaliwal R, Drover JW, Gramlich L, Dodek P: Canadian clinical practice guidelines for nutrition support in mechanically ventilated, critically ill adult patients. JPEN J Parenter Enteral Nutr 2003, 27:355-373.

5. Singer P, Berger MM, Van den Berghe G, Biolo G, Calder P, Forbes A, Griffiths R, Kreyman G, Leverve X, Pichard C, ESPEN: ESPEN Guidelines on Parenteral Nutrition: intensive care. Clin Nutr 2009, 28:387-400.

6. Martindale RG, McClave SA, Vanek VW, McCarthy M, Roberts P, Taylor B, Ochoa JB, Napolitano L, Cresci G: Guidelines for the provision and assessment of nutrition support therapy in the adult critically ill patient: Society of Critical Care Medicine and American Society for Parenteral and Enteral
Nutrition: Executive Summary. Crit Care Med 2009, 37:1757-1761.

7. Marik PE, Zaloga GP: Gastric versus post-pyloric feeding: a systematic review. Crit Care 2003, 7:R46-51

8. Jeffery KM, Harkins B, Cresci GA, Martindale RG: The clear liquid diet is no longer a necessity in the routine postoperative management of surgical patients. Am Surg 1996, 62:167-170.

9. Marik PE, Pinsky M: Death by parenteral nutrition. Intensive care medicine 2003, 29:867-869.

10. Casaer MP, Mesotten D, Hermans G, Wouters PJ, Schetz M, Meyfroidt G, Van Cromphaut S, Ingels C, Meersseman P, Muller J, Vlasselaers D, Debaveye Y, Desmet L, Dubois J, Van Assche A, Vanderheyden S, Wilmer A, Van den Berghe G: Early versus late parenteral nutrition in critically ill adults. N Engl J Med 2011, 365:506-517.

doi:10.1186/cc10591

Cite this article as: Cove ME, Pinsky MR: Early or Late Parenteral Nutrition: ASPEN vs ESPEN. Critical Care 2011, 15:317. 